2015-05

Long-term patterns in interpersonal behaviour amongst psychopathic patients in secure inpatient treatment: $A$ follow-up study.

\title{
Draycott, S
}

http://hdl.handle.net/10026.1/3543

10.1002/pmh.1292

Personal Ment Health

All content in PEARL is protected by copyright law. Author manuscripts are made available in accordance with publisher policies. Please cite only the published version using the details provided on the item record or document. In the absence of an open licence (e.g. Creative Commons), permissions for further reuse of content should be sought from the publisher or author. 
Personality and Mental Health

9: 124-132 (2015)

Published online 26 January 2015 in Wiley Online Library

(wileyonlinelibrary.com) DOI 10.1002/pmh.1292

\section{Long-term patterns in interpersonal behaviour amongst psychopathic patients in secure inpatient treatment: A follow-up study}

SIMON DRAYCOTT ${ }^{1}$, ROXANNA SHORT ${ }^{2}$ AND TIM KIRKPATRICK ${ }^{3},{ }^{1}$ School of Psychology, University of Surrey, Guildford, UK; ${ }^{2}$ School of Psychology, University of Southampton, Southampton, UK; ${ }^{3}$ Peninsula Schools of Medicine and Dentistry, Plymouth University, Plymouth, UK

\section{ABSTRACT}

Psychopathy is a disorder that is partly defined by with maladaptive interpersonal behaviour and has significant effects on treatment outcomes. A previous study (Draycott et al., 2011) found that higher levels of psychopathy led to a specific interpersonal 'trajectory' amongst patients in a secure psychiatric treatment programme during the first 9 months of their admission. In that programme, more psychopathic patients became increasingly dominant over time, and less psychopathic patients became increasingly hostile. This study is a longer-term follow-up and extension of that study, extending the window of observation to 33 months of treatment. It was found that the more psychopathic patients' increased dominance returned to baseline levels by 33 months, as did the less psychopathic patients' increased hostility. This suggests that treatment for this group is not idiopathic but leaves unanswered the question as to what these divergent trajectories represent. Copyright $@ 2015$ John Wiley $\mathcal{E}$ Sons, Ltd.

Psychopathy is a disorder whose effects are disproportionate to its prevalence. Although individuals who could be described as psychopathic are estimated to form about $1 \%$ of the population (Blair, Mitchell \& Blair, 2005), they commit between $30 \%$ and $50 \%$ of all violent crimes (Hare, 1993, 1996, 1999; Hare \& McPherson, 1984). Psychopathic individuals are also up to five times more likely to recidivate than non-psychopathic offenders (Serin \& Amos, 1995). As such, psychopathy has received a great deal of clinical and research attention in an effort to understand its presentation and development in affected individuals. In comparison, relatively little research has focused on attempts to intervene with psychopathic individuals, in part because of an attitude of therapeutic nihilism, that is, that psychopathic offenders are 'untreatable'.

Reidy, Kearns and DeGue (2013) provide a helpful framework for thinking about research into interventions with psychopathic offenders. In particular, much of the available literature does not directly address the question of whether treatment improves, worsens or has no effect on violence committed by psychopathic offenders. Rather, many studies address the question of whether the effectiveness of interventions for offenders is moderated by the presence of psychopathy, i.e. whether psychopaths do worse in treatment than non-psychopaths.

Amongst general forensic samples, including forensic psychiatric samples, psychopathy has very 
often been found to be related to poorer treatment outcomes in terms of both proximal markers for risk and violent recidivism (Olver et al., 2013; Spain et al., 2004). In relation to sexual offenders, psychopathy has been shown to be particularly inimical to positive treatment outcomes. It has been shown to relate to higher rates of treatment dropout and faster sexual and violent recidivism (Barbaree, 2005; Gretton et al., 2001; Hildebrand et al., 2004; Langton et al., 2006; Looman et al., 2005; Olver \& Wong, 2006, 2009). Most worryingly, those psychopathic individuals who appeared to be doing better in treatment were in one study found to be more likely to recidivate than those who were rated as having poorer treatment behaviour (Seto \& Barbaree, 1999). It is on the basis of these studies that an attitude of nihilism about the treatment of psychopathic offenders is often based. However, what all these studies have demonstrated is not whether psychopathic offenders can respond positively to treatment. Rather, they have demonstrated that psychopathic offenders respond more poorly to 'standard' offence-focused interventions than do non-psychopathic offenders, i.e. that psychopathy moderates the effectiveness of non-specialist treatment for offending behaviour.

To date, only a handful of studies have directly addressed the question of whether psychopathy per se can respond to treatment. Rice et al. (1992) reported that violent recidivism for psychopathic men treated in a therapeutic community was higher than violent recidivism for untreated psychopathic men. However, this study has famously been criticized because of the questionable nature of the treatment being applied, which involved such outre methods as marathon nude encounter sessions. The method of defining psychopathy also differed from the current gold standard Psychopathy Checklist-Revised (PCL-R), such that the comparability of the psychopathic group to modern standards may be questionable. More recently, a treatment programme specifically designed for male psychopathic adolescents has been successful in reducing violent recidivism. Psychopathic offenders treated in this programme were less likely to recidivate than equally psychopathic offenders under 'treatment as usual' (Caldwell et al., 2006; Caldwell, 2011) such that post-treatment psychopathy was no longer a significant predictor of recidivism (Caldwell et al., 2007). Studies of this type suggest that nihilism is not necessarily warranted, and that with treatment programmes specifically matched to psychopathic offenders' needs, positive outcomes are possible.

Another small strand of research has attempted to assess not whether psychopathic offenders can benefit from treatment but which psychopathic offenders can benefit. Skeem et al. (2002) for instance, found lower rates of violence during treatment amongst psychopathic individuals if they were given more intensive psychiatric care in the community. This raises the prospect that psychopathic individuals can respond to nonspecialist interventions but simply require a higher dosage of treatment. Unfortunately, this study, although interesting, suffers from a number of flaws due to lack of control over allocation of individuals to levels of treatment intensity. Chakhssi et al. (2010) in a forensic psychiatric setting found that there was an increase in violence amongst psychopathic patients over the course of treatment. However, this increase was due to a small minority of the psychopathic sample (approximately $25 \%$ ). This raises the prospect that a more nuanced approach is required, which addresses differential responses of subgroups of psychopathic offenders.

If the state of the literature on the treatment of psychopathic offenders can be summarised as showing a worse response to treatment as usual, but a potential response to specialized treatment, a number of collateral questions can be raised. One of these is whether the differential outcomes from treatment amongst psychopathic offenders are simply because of a need for a higher dose or whether psychopathic offenders have a specific and different response to treatment attempts compared with non-psychopathic offenders. Draycott et al. (2011) reported an attempt to track 
interpersonal behaviour over time for a sample of forensic inpatients with high levels of psychopathy within a complex psychological treatment programme. The main findings in that study were that patients with higher levels of psychopathy as measured by the PCL-R showed increased levels of interpersonal dominance (but not hostility) between baseline measurement and 6 months follow-up. Less psychopathic patients, on the other hand, showed increased levels of interpersonal hostility (but not dominance) over the same period. The two groups therefore followed distinct interpersonal 'trajectories', although both tended towards worse interpersonal functioning. This raises the possibility that the proximal effects of treatment on psychopathic offenders may differ from the effects of treatment on non-psychopathic offenders. Several hypotheses were offered to explain this pattern although clearly, these may not be exhaustive.

(1) Attempts to treat this particular patient group cause deterioration in interpersonal behaviour, the nature of which is moderated by levels of psychopathy. This would suggest that the effect of treatment was iatrogenic on the individuals' interpersonal behaviour, increasing whichever interpersonal tendencies were already problematic for the individual.

(2) Increased interpersonal dominance (or hostility) represents divergent adaptations to a restrictive environment. The more psychopathic individuals accentuated their natural tendency towards dominance, whereas the less psychopathic individuals accentuated their natural tendency towards hostility. This hypothesis differs from the first in positing that changes in interpersonal behaviour are more short-term 'strategic' responses rather than true long-term iatrogenic effects.

(3) The changes in both cases related to the 'end of the honeymoon' over the relatively short period being observed. On admission, both more and less psychopathic patients were attempting to engage in the treatment programme in order to secure their release. However, with time each 'reverted to type'. This suggests that the observed changes would actually represent a continuation of an underlying interpersonal tendency that had been temporarily masked.

A necessary (but not sufficient) piece of information to help disentangle these hypotheses is how these patients' interpersonal trajectories changed over a longer period. Under the first hypothesis, one might expect interpersonal dysfunction to continue to increase along the two trajectories to an asymptote. Hypotheses 2 and 3 might in contrast allow for an improvement following the initial deterioration as individuals shift away from ineffective attempts to control their environment (Hypothesis 2). Hypothesis 3 would be consistent with either an initial deterioration followed by improvement (if treatment is effective) or a deterioration followed by stabilization (if treatment cannot affect the underlying interpersonal tendencies).

In relation to our understanding of the moderating effect of psychopathy on treatment outcome for offenders, a longer-term follow-up will go a small step towards understanding the negative effects of psychopathy on treatment. If hypothesis 1 is supported, this would add to the literature suggesting that the negative outcome of treatment of psychopathic offenders with non-specialist programmes is iatrogenic. Hypotheses 2 and 3, on the other hand, would still allow that psychopathic offenders might respond to non-specialist treatment, simply taking a different path for improvement and requiring a higher dosage of treatment.

This report focuses on such a longer-term follow-up of the patient group described by Draycott et al. (2011), extending the period of study to 33 months and including four points of assessment. As in the work by Draycott et al. (2011), the data on which this analysis is based are drawn from a routine outcomes audit carried out within one hospital-based Dangerous and 
Severe Personality Disorder treatment programme. This project was managed and overseen by local clinical audit procedures.

\section{Methodology}

\section{Patient characteristics}

Participants for the overall study were drawn from 59 consecutive admissions to a high secure, hospital-based personality disorder service in the UK. All patients were men between the ages of 20 and 65 years, admitted from either the prison service or secure NHS facilities and had committed serious sexual and/or violent offences. All patients within this cohort were assessed using the PCL-R (Hare, 2003). From the possible sample of 59 patients, 38 had completed data on psychopathy and interpersonal behaviour at baseline. Of these, 28 had complete data at 33 months and were selected for this analysis. The remaining 10 patients were still in the service at this point, but their admissions had not yet lasted for 33 months. The group with complete data had an average PCL-R score of 26.25 with a standard deviation of 5.82. For comparison, the patients who did not have complete data at 33 months had an average PCL-R score of 26.22 with a standard deviation of 4.99. There were no significant differences between these two groups on this measure $(t=-0.13$, degrees of freedom (d.f. $)=35$, $p=0.99)$.

The initial scores on the main outcomes measures of interest here (the Warmth and Dominance indices of the Chart of Interpersonal Relations in a Close Living Environment, described in the succeeding text) were 0.53 on the Warmth index and 0.33 on the Dominance index. The corresponding scores for the patients who did not have complete data at 33 months were -0.19 and 0.53 . There is therefore a slight but nonsignificant difference between these groups on the Warmth index $(t=-1.75$, d.f. $=35, p=0.09)$ and no significant differences on the Dominance index $(t=0.65$, d.f. $=35, p=0.52)$. There are some indications therefore that the subgroup of patients whose data were analysed here was somewhat less hostile than the subgroup who did not have data available at 33 months.

A median split was used to define high and low psychopathy groups; those assigned to the 'high' group (14 patients) had PCL-R scores equal to or greater than 28 . It should be noted that this represents a skewed sample compared with the general UK prison population, with a high representation from those with greater levels of psychopathy and a low representation from those with minimal levels of psychopathy.

\section{Description of the treatment programme}

Within the service, formal therapy was delivered in small groups that focused on educating patients about cognitive-behavioural models of their disorders and behavioural patterns, developing selfmonitoring and insight and learning new skills to challenge dysfunctional patterns of thinking, feeling and behaving. These groups are embedded in a framework that matches the interventions to the patient's 'stage of change' (Prochaska \& DiClemente, 1983) with those who did not yet recognize that they had any 'problems' undertaking work focusing on motivation, goal-setting and self-management and those who had accepted that they had treatment needs undertaking offence-focused programmes. Individuals' levels of engagement in treatment varied widely: some engaged in the full range of available treatments, whereas others refused to engage in any therapeutic activities. In order to assess for potential systematic biases, all patients in this sample were categorized as treatment refusers (did not take part in any of the psychological therapies on offer), partial engagers (took part in some recommended therapies but refused one or more other recommended therapies) or full engagers (took part in all recommended therapies). This categorization was carried out on the basis of examination of their file notes by the main author. There were no significant differences between the high and 
low psychopathy groups in terms of the therapy engagement by this rubric (Somers' $d=-0.57$, $p=0.72$ ), although given the low $N$ at each level of engagement, this comparison does lack power.

This study forms part of an ongoing evaluation of this service as part of its clinical governance commitments. Data were collected as part of routine clinical practice and used for feedback on progress in treatment to individual patients and their clinical teams. These data were also collated in an anonymized form on a regular basis and used to evaluate the effectiveness of the service.

\section{Measures}

The interpersonal behaviour of these patients was assessed using the Chart of Interpersonal Reactions in Closed Living Environments (CIRCLE; Blackburn and Renwick, 1996). The CIRCLE is a 51-item (each rated on a 4-point Likert scale) observer-rated measure of interpersonal behaviour that is suitable for use within forensic psychiatric environments. Scores are produced for each octant of the interpersonal CIRCLE, which can then be used to derive a placement for the individual on the Warmth and Dominance axes. Inter-rater reliability for some of the octants is moderate (Blackburn \& Renwick, 1996), and to overcome this, it is recommended that the measure is completed by two staff members, and their ratings averaged for each item. CIRCLE items were completed by nursing staff who knew the patient well on the basis of their observations of that individual's interpersonal behaviour.

As the Dangerous and Severe Personality Disorder service was intended to assess and treat patients with high levels of personality disorder and psychopathy, all patients within the service were routinely assessed with a number of standardized personality disorder and risk measures. Amongst these was the Hare PCL-R (Hare, 2003). The PCL- $R$ is a 20-item scale for the assessment of psychopathy in research, clinical and forensic settings. It uses a semi-structured interview alongside file and collateral information to measure inferred personality traits and behaviours related to the concept of Psychopathy. The PCL-R provides a reliable and valid assessment of the lifetime presence and severity of symptoms of psychopathic personality disorder, and it is widely considered to be the 'gold standard' assessment of psychopathy in forensic settings. Evaluation of the PCL-R has suggested a variety of factor solutions (Hare $\&$ Neumann, 2006). However, a consistent theme is that of two factors relating to a cold, callous interpersonal style and an impulsive, antisocial lifestyle respectively. These factors have been found to be highly correlated in most samples (Hare \& Neumann, 2006).

\section{Procedure}

All patients within the service were assessed using the CIRCLE at 3 months after their admission to the service. This coincided with their first Care Programme Approach meeting and allowed sufficient time for staff to observe them so that they were able to comment accurately on their behaviour. Two staff members who worked closely with the patient were asked to independently complete the CIRCLE, focusing on the patient's behaviour over the previous month. After further 6 months, at the patient's second Care Programme Approach, two staff members were again asked to complete the CIRCLE. Subsequent assessments were completed yearly for all patients. As such, after 33 months of admission, patients had four data points available.

Given staff turnover and patient movement, it was not always possible for the same staff members who completed the first CIRCLE assessment to complete the subsequent assessments. This introduced an extra and unpredictable element of error into the ratings of interpersonal behaviour. It should also be noted that all assessments were made at the same time points relative to the patients' admission dates, and therefore, they were not conducted on the same dates for all patients. There were significant changes to the service over the course of these assessments, including the 
opening of a permanent, purpose-built building. These changes will not have affected all patients equally.

\section{Analysis}

A $2 \times 2 \times 4$ repeated measures ANOVA was used to assess for the effects of high vs. low psychopathy (the between subjects factor of Psychopathy) on the orthogonal measures of Warmth and Dominance (the within-subjects factor of Dimension) over four time points. Where significant effects were found in omnibus tests, these were then followed up with one-way ANOVAs and paired t-tests for the high vs. low psychopathy groups separately and with independent $t$-tests to compare the high and low psychopathy groups at each time point.

\section{Results}

The omnibus ANOVA highlighted a significant interaction of Dimension and Psychopathy $(F=4.21, p=0.05)$, as well as a significant interaction of Dimension and Time $(F=3.01, p=0.04)$. Tests of within-subjects contrasts suggested that this latter relationship was significant specifically at the quadratic level $(F=8.21, p=0.01)$. These contrasts also highlighted a significant quadratic relationship between Psychopathy and Time $(F=4.94, p=0.04)$. To further analyse these relationships, separate repeated measures ANOVAs were carried out for the dimensions of Warmth and Dominance separately across the four time points.

For Warmth, the overall ANOVA did not highlight any significant main effects or interactions, although the main effect of Time trended towards significance $(F=2.48, p=0.07)$. Withinsubjects contrasts, however, highlighted a significant quadratic effect of Time $(F=9.13, p=0.01)$. Paired $t$-tests looking at all combinations of Time, collapsed across Psychopathy, showed a significant difference in Warmth between the first and second time points, i.e. between 3 months post-admission and 9 months post-admission $(t=2.02, p=0.05)$. A significant difference was also highlighted between the first and third time points, i.e. between 3 months post-admission and 21 months postadmission $(t=2.24, p=0.03)$. No other comparisons were statistically significant. This suggests that with respect to the interpersonal dimension of Warmth, there were no significant effects of Psychopathy in this group. There appears to have been a curvilinear change in Warmth over the course of admission, with an initial decrease followed by a steady increase back to baseline levels. These relationships are summarised in Figure 1.

With respect to Dominance, the overall ANOVA did not highlight any significant linear or quadratic effects of Psychopathy but did highlight the significant main effect of Psychopathy $(F=4.53, p=0.04)$. Because of this, the effects of Time were examined for each level of Psychopathy separately with separate repeated measure ANOVAs. For the low psychopathy group, there were no significant linear or quadratic effects of Time. For the high psychopathy group, there was a significant quadratic (but not linear) effect of time $(F=4.90, p=0.04)$.

These effects were further decomposed using paired $t$-tests for all combinations of time points carried out for each level of Psychopathy separately. For the low psychopathy group, there were

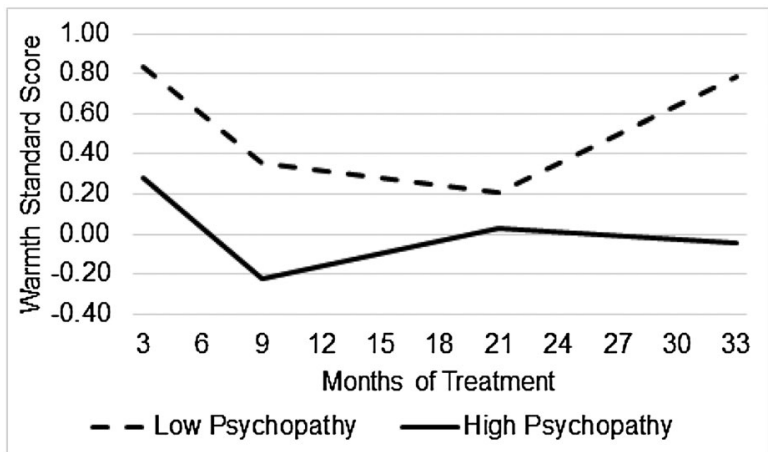

Figure 1: Summary of changes in interpersonal warmth for low and high psychopathy patients over four time points ( 1 = 3 months, 2 =9 months, $3=21$ months, $4=33$ months $)$ 


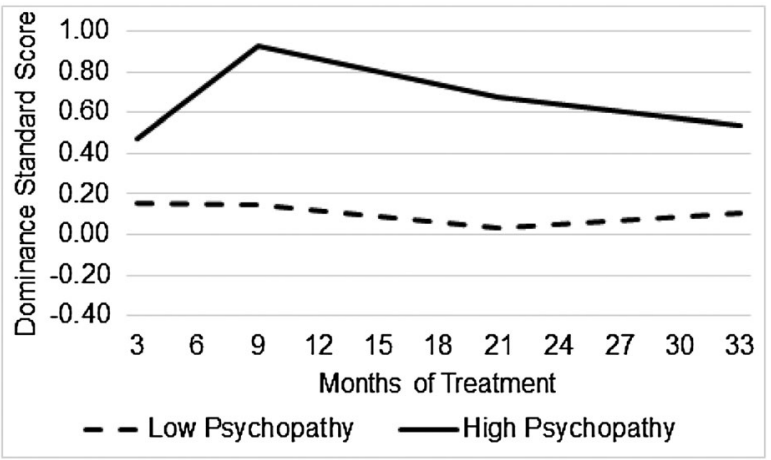

Figure 2: Summary of changes in interpersonal dominance for low and high psychopathy patients over four time points ( 1 = 3 months, 2 =9 months, $3=21$ months, 4 = 33 months $)$

no significant differences in Dominance between any time points. For the high psychopathy group, there was a significant difference in Dominance between the first and second time points, i.e. between 3 months post-admission and 9 months post-admission $(t=-2.90, p=0.01)$. However, there were no other significant differences. Finally, independent $t$-tests were carried out comparing the low and high psychopathy groups on Dominance at each time point. This showed a significant difference between the two groups only at the second time point, i.e. 9 months postadmission $(t=-2.42, p=-0.02)$. These results suggest a curvilinear change in Dominance over time, which was specific to the higher psychopathy patients, with an initial marked increase in Dominance followed by a fall back to baseline levels by 33 months. These relationships are summarised in Figure 2.

\section{Discussion}

The main aim of this analysis was to rule out the hypothesis that previously observed changes in interpersonal behaviour in psychopathic inpatients that represented a permanent iatrogenic effect of psychiatric admission. In this respect, this goal was achieved. The increased interpersonal dominance exhibited by these patients reduced to baseline levels over 33 months. Similarly, the more general decrease in warmth (or increase in hostility) amongst these patients returned to baseline after 33 months. We can therefore conclude that whatever else is happening amongst this sample, psychiatric admission does not make these patients' behaviour worse in the medium term. Conclusions cannot of course be drawn from this about their likely behaviour in the long term or post-discharge.

This analysis also appears to confirm a specific interpersonal trajectory for more psychopathic inpatients. In general, in this sample, such patients were more interpersonally dominant, and they also showed a change in this behaviour that followed an inverted U over the course of admission. The reasons behind this specific trajectory remain to be elucidated, but one hypothesis is that psychopathic patients' deficiencies in affect towards others lead them to interpret interpersonal relationships in a more unidimensional manner, paying more attention to aspects of dominancesubmission than to warmth-hostility. When under pressure to conform in a restrictive environment, they rely again on this unidimensional view of relationships, 'upping the ante' in their interpersonal dominance.

There are multiple reasons why caution should be exercised about these conclusions, such that it cannot be stated that this trajectory should be expected for all psychopathic individuals in treatment. This sample is both small and highly selected, chosen specifically to have higher levels of psychopathy than would be expected amongst UK forensic samples generally. The 'low' psychopathy group is therefore equivalent to a 'moderate' psychopathy group in most of the literature, and the true interpersonal trajectory of low psychopathy inpatients in forensic settings has not been assessed here. The PCL-R assessments carried out here were also performed as part of routine clinical practice and as such have not been subjected to the types of quality control efforts such as assessment of inter-rater reliability. It is also the case that the level of treatment received by patients within this sample varied greatly, from outright 
refusal to engage to complete compliance with all treatment. Although there were no gross differences between the high and low psychopathy groups in terms of the numbers of patients refusing, partially engaging or fully engaging in treatment, this does not rule out the possibility of more subtle and systematic differences on how the two groups engaged (or not) in the treatment programme. Additional (and possibly systematic) error is introduced by the lack of consistency on who provided the ratings of interpersonal behaviour over time. Another possibility is that the apparent shifts in interpersonal dominance and warmth simply reflect staff 'normalizing' patients' interpersonal behaviour and therefore rating them as less hostile or dominant. This latter possibility is unlikely, given the changes on who was rating the patient over time and the specific trajectories found (i.e. an initial worsening before improvement rather than a simple linear change). However, it cannot be entirely ruled out given the nature of this data set.

This analysis is however strengthened by several features. Principle amongst these is its ecological validity, i.e. the degree to which the conditions of the study can be generalized to 'real world' conditions. Patients were observed under 'natural' circumstances rather than in an idealized environment. This study also provides follow-up over an extended period, allowing the examination of changes that took place only over the course of many months. Studies that examine changes only over the course of 6 or even 12 months with this patient group might have erroneously concluded that a negative response to treatment was purely iatrogenic.

In contrast to the more pessimistic results in the treatment literature on psychopathy, this study suggests that treatment does not necessarily make psychopaths worse. However, it is not possible on the basis of these data to state that treatment was beneficial for these patients either. At best, the pattern shows a worsening followed by a return to baseline. From our initial three hypotheses, two now remain. The first is broadly 'pessimistic', suggesting that the psychopathic patients' behaviour genuinely deteriorated at the beginning of the admission, before slowly returning to its starting point over a protracted period. This pessimistic hypothesis would argue that active attempts to treat psychopaths are hardly cost-effective.

The second hypothesis is more 'optimistic' and holds that the initial appearance of relatively 'good' behaviour at the start of admission was due to a 'honeymoon period'. During this period, patients of all types attempt to present themselves in the best possible light so as to reduce the duration of their admission. Over the first few months, frustration sets in and these attempts are abandoned, leading to a worsening of behaviour. In this hypothesis, the later reduction in hostile behaviour (across all patients) or dominant behaviour (across only psychopathic patients) would represent a genuine and positive change. This hypothesis would suggest that psychopaths are capable of responding to non-specialist treatment, once an initial 'burst' of increased interpersonal dominance is overcome. However, if treatment is terminated during this 'burst', the effect might well be a deterioration of behaviour relative to non-psychopathic offenders in the same treatment programme.

Unfortunately, it is not possible on the basis of these data to differentiate between these two hypotheses. This would require data on interpersonal behaviour collected prior to admission. There would also be utility in gathering data further into admission, to see if interpersonal behaviour continued to improve to a point beyond the patients' baseline behaviour or stabilized at their baseline. Unfortunately, because of the closure of this treatment facility, and with it the evaluation from which these data were drawn, it will not be possible to pursue either of these options within this patient group. Both options should be explored in future research. It is also recommended that these differing interpersonal trajectories in treatment are more directly linked to therapy outcomes, for instance, by comparing them to proximal risk assessment measures or to ultimate reoffending. If it were to be found that a particular intepersonal trajectory was both more common in highly psychopathic offenders and had an association with poor treatment outcome, this 
could help to clarify the mechanism through which psychopathy interferes with treatment progress.

\section{References}

Barbaree, H. E. (2005). Psychopathy, treatment behavior, and recidivism: An extended follow-up of Seto and Barbaree. Journal of Interpersonal Violence, 20, 1115-1131.

Blackburn, R., \& Renwick, S. J. (1996). Rating scales for measuring the interpersonal circle in forensic psychiatric patients. Psychological Assessment, 8(1), 76-84.

Blair, J., Mitchell, D., \& Blair, K. (2005). The psychopath: Emotion and the brain. Malden: Blackwell Publishing.

Caldwell, M. F. (2011). Treatment related changes in behavioral outcomes of psychopathy facets in adolescent offenders. Law and Human Behavior, 35, 275-287.

Caldwell, M. F., McCormick, D. J., Umstead, D., \& Van Rybroek, G. J. (2007). Evidence of treatment progress and therapeutic outcomes among adolescents with psychopathic features. Criminal Justice 83 Behavior, 34, 573-587.

Caldwell, M. F., Vitacco, M., \& Van Rybroek, G. J. (2006). Are violent delinquent worth treating? A cost-benefit analysis. Journal of Research in Crime EO Delinquency, 43, 148-168.

Chakhssi, F., de Ruiter, C., \& Bernstein, D. (2010). Change during forensic treatment in psychopathic versus nonpsychopathic offenders. The Journal of Forensic Psychiatry 8 Psychology, 21, 660-682.

Draycott, S., Askari, R., \& Kirkpatrick, T. (2011). Patterns and change in psychopathic interpersonal behaviour in forensic inpatient treatment. Personality and Mental Health, 5(3), 200-208.

Gretton, H. M., McBride, M., Hare, R. D., O'Shaughnessy, R., \& Kumka, G. (2001). Psychopathy and recidivism in adolescent sex offenders. Criminal Justice 8 Behavior, 28, 427-449.

Hare, R. D. (1993). Without conscience: The disturbing world of the psychopaths among us. New York, NY: Simon \& Schuster.

Hare, R. D. (1996). Psychopathy: A clinical construct whose time has come. Criminal Justice and Behavior, 23(1), 25-54.

Hare, R. D. (1999). Psychopathy as a risk factor for violence. Psychiatric Quarterly, 70(3), 181-197

Hare, R. D. (2003). Manual for the revised psychopathy checklist (2nd ed.). Toronto, ON, Canada: Multi-Health Systems.

Hare, R., \& McPherson, L. (1984). Violent and aggressive behaviour by criminal psychopaths. International Journal of Law and Psychiatry, 7(1), 3-50.

Hare, R. D., \& Neumann, C. S. (2006). The PCL-R assessment of psychopathy: Development, structural properties, and new directions. In C. Patrick (Ed.), Handbook of psychopathy (pp. 58-88). New York: Guilford Press.

Hildebrand, M., de Ruiter, C., \& de Vogel, V. (2004). Psychopathy and sexual deviance in treated rapists:
Association with sexual and nonsexual recidivism. Sexual Abuse: A Journal of Research 8 Treatment, 16, 1-24.

Langton, C. M., Barbaree, H. E., Harkins, L., \& Peacock, E. J. (2006). Sex offenders' response to treatment and its association with recidivism as a function of psychopathy. Sexual Abuse: A Journal of Research Eु Treatment, 18, 99-120.

Looman, J., Abracen, J., Serin, R., \& Marquis, P. (2005). Psychopathy, treatment change, and recidivism in high-risk, high-need sexual offenders. Journal of Interpersonal Violence, 20(5), 549-568.

Olver, M., \& Wong, S. (2006). Psychopathy, sexual deviance, and recidivism among sex offenders. Sexual Abuse: A Journal of Research Eु Treatment, 18, 65-82.

Olver, M., \& Wong, S. (2009). Therapeutic responses of psychopathic sexual offenders: Treatment attrition, therapeutic change, and long-term recidivism. Journal of Consulting and Clinical Psychology, 77, 328-336.

Olver, M., Lewis, K, \& Wong, S. (2013). Risk reduction treatment of high-risk psychopathic offenders: The relationship of psychopathy and treatment change to violent recidivism. Personality DisordersL Theory, Research and Treatment, 4(2), 160-167.

Prochaska, J. O., \& DiClemente, C. C. (1983). Stages and processes of self-change of smoking: Toward an integrative model of change. Journal of Consulting and Clinical Psychology, 51(3), 390-395.

Reidy, D., Kearns, M., \& DeGue, S. (2013). Reducing psychopathic violence: A review of the literature. Aggression and Violent Behavior, 18(5), 527-538.

Rice, M. E., Harris, G. T., \& Cormier, C. A. (1992). An evaluation of a maximum security therapeutic community for psychopaths and other mentally disordered offenders. Journal Law and Human Behavior, 16(4), 399-412.

Serin, R., \& Amos, N. (1995). The role of psychopathy in the assessment of dangerousness. International Journal of Law and Psychiatry, 18(2), 231-238.

Seto, M. C., \& Barbaree, H. E. (1999). Psychopathy, treatment behavior and sex offender recidivism. Journal of Interpersonal Violence, 14(12), 1235-1248.

Skeem, J. L., Monahan, J., \& Mulvey, E. P. (2002). Psychopathy, treatment involvement, and subsequent violence among civil psychiatric patients. Law and Human Behavior, 26, 577-603.

Spain, S. E., Douglas, K. S., Poythress, N. G., \& Epstein, M. (2004). The relationship between psychopathic features, violence and treatment outcome: The comparison of three youth measures of psychopathic features. Behavioral Sciences 8 the Law, 22, 85-102.

Address correspondence to: Simon Draycott, School of Psychology, Faculty of Arts and Human Sciences, University of Surrey, Guildford GU2 7XH, UK. Email: s.draycott@surrey.ac.uk 